\title{
Estimation of English Proficiency Based on Read-Aloud Performance and Linguistic Difficulty of Sentences
}

\author{
Katsunori Kotani* and Takehiko Yoshimi**
}

(Received 4 April 2021 and accepted in revised form 14 September 2021)

\begin{abstract}
In this study, the English proficiency (EP) of learners was determined based on their read-aloud performance (RAP). RAP reflects EP as it demonstrates the basic linguistic skills required for recognizing lexical/syntactic structure, understanding the meaning, and making speech sounds depending on the sentence structure/meaning. Empirically, RAP test scores have been correlated with those of EP tests such as the Test of English as a Foreign Language or Test of English for International Communication. With the assumption that RAP can be evaluated based on accuracy, speech rate, and learners' subjective judgment, in this study, it was examined which combinations of these RAP indices achieved the highest EP estimation; in addition, how EP estimation depended on learners' EP was analyzed. For this, EP estimation was evaluated for learners at different EP levels. The experimental results showed that all combinations of RAP indices perform comparably in EP estimation, and that effective EP estimation differed by EP level.
\end{abstract}

Keywords English as a second language, proficiency measurement, read-aloud performance, regression, proficiency level

\section{Introduction}

Evaluating English proficiency (EP) is an essential task for teachers of English as a second language (ESL). EP refers to learners' English language proficiency, which includes listening, speaking, reading, and writing skills. One of the goals behind evaluating EP is to investigate how learners' proficiency increases throughout the course. For example, this can be examined through classroom-based assessment (CBA) - a read-aloud performance (RAP) test can be conducted for CBA. EP can be evaluated through tests such as the Test of English as a Foreign Language (TOEFL) and/or Test of English for International Communication (TOEIC).

While EP tests reduce ESL teachers' time and effort required for preparing course-centered exams, the former have a few limitations: fees are high and duration is long-for example, TOEFL and TOEIC require more than two hours; additionally, the text content is irrelevant to ESL classes.

To overcome these limitations, evaluating RAP is acceptable on conceptual and empirical grounds. Conceptually, RAP demonstrates how well learners

\footnotetext{
* College of International Professional Development, Kansai Gaidai University, Japan

** Faculty of Advanced Science and Technology, Ryukoku University, Japan
}

use basic linguistic skills to recognize lexical/syntactic structure, understand the meaning of the word/sentence, and make speech sounds depending on sentence structure/meaning. Empirically, read-aloud tasks have been used as items in recent computer-based EP tests on applications such as Duolingo and Versant.

The RAP test is a promising CBA method. Previous research on $\mathrm{CBA}^{[1]}$ has revealed that a readaloud task is a reliable test item in CBA $(\alpha=0.86)$. See Grabe and $\mathrm{Jian}^{[2]}$ for a brief review of the advantages of CBA through a RAP test.

Kotani and Yoshimi ${ }^{[3]}$ measured RAP through the following three indices: accuracy, speech rate, and learners' subjective judgment of the ease of reading aloud-however, they did not examine which combination of these indices would be the most effective for evaluating RAP. To measure learners' EP, fewer RAP indices should be used; since RAP tests with fewer indices require less time to prepare and incur a lower cost, these are more practical and useful.

Lee $^{[4]}$ observed that the correlation between speech rate and oral EP seemed to be dependent on the learners' EP level, but this dependency was not examined in detail. If such a dependency is observed, the use of EP estimation would be limited to learners with levels appropriate for it - in other words, their EP would have to be confirmed beforehand. However, if EP estimation is independent of learners' EP, it could be ascertained without 
confirming the latter. Furthermore, EP estimation can be used as a placement test to determine learners' EP level.

This study was conducted to investigate whether ESL teachers can develop an EP estimation method for learners in their classes - to this end, the practical applicability of EP estimation was examined to answer the following research questions (RQs):

RQ1: What combinations of the RAP indices accuracy, speech rate, and ease of reading aloud can achieve the highest EP estimation?

RQ2: How is EP estimation dependent on learners' EP?

\section{Collection of Read-Aloud Data}

Training data for EP estimation were compiled from 50 participants: 16 at the beginner level, 16 at the intermediate level, and 18 at the advanced level. The use of small-size training data reveals the possibility of ESL teachers developing an EP estimation method based on such data compiled in their classes.

The read-aloud data included 50 instances in which the participants read the given material aloud. The data used comprised speech sound, three types of RAP scores, linguistic features of the read-aloud material, and the participants' test scores for EP.

\subsection{Participants}

The participants were English learners, and their first language was Japanese. They were students of universities in Tokyo, Japan (28 men and 22 women) with a mean age of 20.8 years and a standard deviation $(S D)$ of 1.3 . They were given monetary compensation for participating in the study.

\subsection{Data Collection Procedures}

The read-aloud task was performed as follows: First, the participants listened to a reference speech sound by a native speaker of American English, who was a voice actor (female, 35 years old). Subsequently, they judged their own performance after reading aloud. Their recording durations were noted to calculate the speech rates.

The participants were given three instructions: 1) read each sentence twice if necessary; 2) complete each task at a speed you are comfortable with; and 3) do not read too fast or too slow, or return and revise a sentence after having moved on to the next one.

\subsection{Read-Aloud Material}

The read-aloud material comprised two texts, one by the International Phonetic Association ${ }^{[5]}$ and the other by Deterding ${ }^{[6]}$. As these texts included basic English sounds, an analysis of the read aloud could reveal the types of English sounds that influence English learners' pronunciation.

The texts featured two Aesop's Fables: The North Wind and the Sun (Text I) and The Boy Who Cried Wolf (Text II). Text I contained five sentences, and Text II contained 10. It should be noted that Text I accidentally failed to include certain sounds such as the initial and medial /z/ and syllable initial / $\theta /$; however, Text II had these.

\subsection{Determining EP}

EP was determined using the participants' TOEIC listening and reading test scores of the current or previous year. The test has a strong correlation with the Language Proficiency Interview of the Foreign Service Institute of the U.S. Department of State ${ }^{[7]}$ and does not have a read-aloud section.

\section{Features for Regression}

In this study, EP was estimated through regression based on RAP scores and linguistic features of a sentence. Support vector machines ${ }^{[8]}$ were used to deal with the machine learning-related problem of small-sized training data lowering the performance of machine learning algorithms. The use of these machines is an advantage of this study as they have a higher regression performance than multiple linear regression analysis; they have also been adopted in previous research ${ }^{[3]}$ to estimate EP.

\subsection{RAP}

RAP was evaluated through three indices: subjective judgment of the ease of reading aloud (EASE), readaloud accuracy (ACC), and speech rate in words per minute (RATE).

EASE was determined through each participant's subjective judgment on a five-point Likert scale $(1=$ difficult, $2=$ somewhat difficult, 3 =average, $4=$ somewhat easy, and 5=easy) - the higher the score, the easier the participant considered the texts.

ACC was calculated by dividing the number of 
words read aloud correctly by the number of words in the corresponding sentence ( 0 indicated that not a single word was read aloud correctly and 1 indicated that all were). A native English speaker evaluated the participants' RAP word by word, but not phoneme by phoneme, using binary decision (correct or incorrect pronunciation). The evaluator was trained to replicate business meetings and interviews in conversation, but was not familiar with the English spoken by the participants. Before the evaluation, the evaluator read the reference texts.

RATE was calculated by dividing the number of words by the time taken to read aloud.

The RAP scores were determined by calculating the mean EASE/ACC/RATE in the 15 sentences.

\subsection{Linguistic Features}

In this study, sentence length, mean word length, number of multiple-syllable words, and word difficulty were the linguistic features considered - these were automatically derived from the 15 sentences.

Sentence length ${ }^{[9]}$ was defined as the number of words in a sentence.

Mean word length ${ }^{[9]}$ was derived by dividing the number of syllables by the number of words in the sentence. The number of syllables in a word ${ }^{[10]}$ was counted using the following steps: count the total number of vowels in the word, subtract any silent vowels, and subtract one vowel from every diphthong.

The number of multiple-syllable words in a sentence ${ }^{[11]}$ was derived using the formula $\sum_{(i=1)}^{N}\left(S_{i}-1\right)$, where $N$ denotes the number of words in the sentence and $S_{i}$ denotes the number of syllables in the $i$-th word. This formula ignores single-syllable words.

Word difficulty was defined as the percentage of words not in the "List of 718 Basic Spoken Words" in relation to the total number of words in the sentence.

These features were calculated for each of the 15 sentences.

\section{Estimation of EP with RAP and Linguistic Features}

Estimation methods were developed using support vector regression, considering EP as the dependent variable and RAP scores and linguistic features as the independent variables.

Support vector regression was conducted using the function "svm( )" defined in the "e1071" package of the software environment $R^{[13]}$. The radial basis function was set as a type of kernel function, and the other parameters of "svm( )" were set as default.

The estimation methods were evaluated using a leave-one-out cross-validation test, considering one instance as test data and $n-1$ instances as training data. Correlation analysis was performed between the estimated and observed EP values. The significance threshold was adjusted for multiple testing $(n=28$, where $n$ is the number of tests performed) based on the false discovery rate $\mathrm{e}^{[14]}$. A statistically significant correlation was further examined for answering the RQs, but a nonsignificant correlation was left out.

To answer RQ1, estimation methods were developed using the linguistic features and seven types of RAP scores as follows: estimation methods using a single index of RAP (EASE or ACC or RATE) or combinations (EASE \& ACC or EASE \& RATE or ACC \& RATE or EASE \& ACC \& RATE). RQ1 was answered by testing the equality of the statistically significant correlation coefficients with the maximum coefficients in chi-square tests. The training/test data included 50 instances.

To answer RQ2, the training/test data were divided into three EP levels according to the Institute for International Business Communication ${ }^{[15]}$ : beginner (below 490, $n=16$, Beg-data), intermediate (490 to 725 , $n=16$, Int-data), and advanced ( 730 or above, $n=18$, Adv-data). Seven types of estimation methods were developed, in addition to those for RQ1. Like RQ1, RQ2 was answered using the chi-square test for the equality of correlation coefficients with the maximum coefficients. Here, a chi-square test was carried out for each data set- that is, Beg-data, Int-data, and Adv-data.

\section{Experimental Results}

\subsection{Descriptive Statistics}

Fig. 1 shows the distribution of EP-normal, according to the Kolmogorov-Smirnov test $(K=0.82, p=.25)$. The mean and minimum and maximum EP values were 607.7, 295, and 900 respectively, and the $S D$ was 186.2.

Table 1 shows the means and $S D$ s of the RAPs of the participants divided thus: the mixed-EP level group (Mix), beginner-level group (Beg), intermediate-level group (Int), and advanced-level group (Adv). As the 
difference between Beg and Int was smaller than that between Int and Adv for EASE, ACC, and RATE, the read-aloud material was considered as easy for the participants. Table 2 shows the means and $S D$ s of the linguistic difficulty of the 15 sentences.

\subsection{Results and Discussion}

Table 3 shows the correlation coefficients between the estimated and observed EP values in the cross-validation tests for the groups. When the correlation coefficient was significantly different from zero, it was marked with an asterisk: those for all RAP indices and their combinations in the Mix-data, those for RATE and three combinations in the Beg-data, and those for indices and combinations barring ACC in the Int-data. No statistically significant correlation was observed in the Adv-data.

Table 3 also shows the results of the chi-square

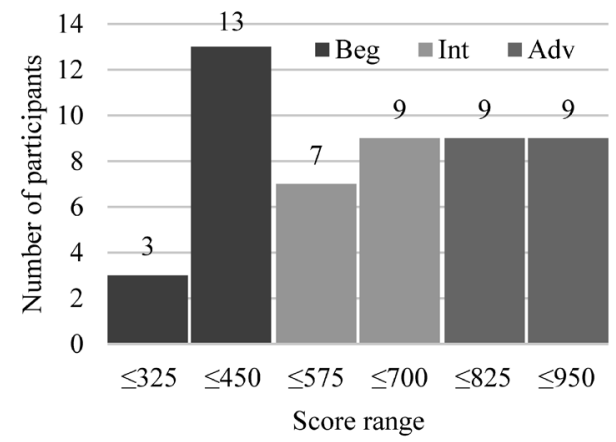

Figure 1. EP distribution

Table 1. Descriptive statistics of RAP: Mean $(S D)$

\begin{tabular}{lcccc}
\hline RAP & $\operatorname{Mix}(n=50)$ & $\operatorname{Beg}(n=16)$ & $\operatorname{Int}(n=16)$ & $\operatorname{Adv}(n=18)$ \\
\hline EASE & $2.97(057)$ & $2.85(0.58)$ & $2.88(0.65)$ & $3.17(0.44)$ \\
ACC & $0.95(0.03)$ & $0.93(0.03)$ & $0.95(0.02)$ & $0.97(0.01)$ \\
RATE & $100.66(15.91)$ & $91.77(13.69)$ & $98.04(13.08)$ & $110.89(14.89)$ \\
\hline
\end{tabular}

Table 2. Descriptive statistics of the linguistic difficulty of the sentences

\begin{tabular}{lrrc}
\hline \multicolumn{1}{c}{ Linguistic features } & $n$ & Mean & \multicolumn{1}{c}{$S D$} \\
\hline Sentence length & 15 & 21.93 & 7.32 \\
Mean word length & 15 & 1.26 & 0.11 \\
Number of multiple-syllable words & 15 & 5.93 & 2.75 \\
Word difficulty & 15 & 0.26 & 0.09 \\
\hline
\end{tabular}

tests for equality of correlation between the maximum correlation coefficient and others - the maximum correlation coefficient for a group is underlined-ACC (Mix-data), EASE \& RATE (Beg-data), and RATE (Int-data); the bold coefficients indicate nonsignificant differences from the maximum coefficients in the group.

RQ1 was answered by the results of the chi-square test for the Mix-data compiled from the participants at different EP levels. The performance of EP estimation $(r=0.32-0.66)$ was not statistically different between the estimation methods using different RAPs. Thus, every combination of the three indices resulted in the most accurate estimation of EP in the mixed-EP group.

RQ2 was answered by the results of the chi-square tests for the Beg-, Int-, and Adv-data. The dependency of EP estimation on EP level was confirmed by the distribution of the correlation coefficient equivalent to the maximum correlation coefficient-that is, the effective estimation method. Effective estimation methods were developed using RATE, EASE \& RATE (max.), ACC \& RATE, and EASE \& ACC \& RATE for the Beg-data and EASE, RATE (max.), EASE \& ACC, EASE \& RATE, ACC \& RATE, and EASE \& ACC \& RATE for the Int-data. For the Adv-data, no effective estimation method was confirmed. Consequently, it was concluded that the effectiveness of EP estimation depended on the EP level. The results of Beg- and Int-data suggest that EP estimation can be developed with training data compiled from at least 16 students.

The generalizability of the results is limited by the uniform distribution of the participants' EP levels and the difficulty of the read-aloud material. Although a uniform distribution is preferable in an experimental design, such distribution might be rare in actual ESL classes, because learners could be classified by their EP level, or because the EP levels could follow a normal

Table 3. Correlation coefficients of RAP for the four groups

\begin{tabular}{lllll}
\hline \multicolumn{1}{c}{ RAP } & Mix & \multicolumn{1}{c}{ Beg } & \multicolumn{1}{c}{ Int } & Adv \\
\hline EASE & $\mathbf{0 . 3 2}^{*}$ & 0.19 & $\mathbf{0 . 6 1} 1^{*}$ & 0.28 \\
ACC & $\underline{0.66^{*}}$ & 0.45 & 0.28 & 0.44 \\
RATE & $\mathbf{0 . 5 3}^{*}$ & $\mathbf{0 . 6 0}^{*}$ & $\underline{0.81^{*}}$ & 0.50 \\
EASE \& ACC & $\mathbf{0 . 3 2}^{*}$ & 0.20 & $\mathbf{0 . 6 1}^{*}$ & 0.28 \\
EASE \& RATE & $\mathbf{0 . 5 3}^{*}$ & $\underline{0.61^{*}}$ & $\mathbf{0 . 8 0}^{*}$ & 0.50 \\
ACC \& RATE & $\mathbf{0 . 5 1}^{*}$ & $\mathbf{0 . 5 7}^{*}$ & $\mathbf{0 . 8 1}^{*}$ & 0.48 \\
EASE \& ACC \& RATE & $\mathbf{0 . 5 2 *}$ & $\mathbf{0 . 5 9}^{*}$ & $\mathbf{0 . 8 0}^{*}$ & 0.47 \\
\hline
\end{tabular}


distribution. As pointed out in Section 5.1, the readaloud material was judged as easy based on EASE, ACC, and RATE - while from the viewpoint of experimental design, easy read-aloud material is preferable, in actual ESL classes, it could be more difficult.

\section{Conclusion}

In this study, EP estimation methods that used different RAP indices and their combinations (EASE, ACC, RATE, EASE \& ACC, EASE \& RATE, ACC \& RATE, and EASE \& ACC \& RATE) were compared and the dependency of EP estimation on EP level (beginner/ intermediate/advanced) examined.

The results indicate that accurate EP estimation was achieved with every RAP indices, and that under the conditions of uniform distribution of EP levels and use of relatively easy read-aloud material, the effectiveness of EP estimation depended on the levels. Additionally, this study indirectly confirmed the applicability of EP estimation, considering that it could be developed with small-sized data $(n=16-50)$. The study also showed the practical limitations of effective EP estimation for learners at different EP levels.

Future research is required to examine the estimation results and improve the estimation method. Methodologically, future research will enable the examination of different estimation models to improve estimation and decrease the cost of preparing supervised training data; pedagogically, it is needed to investigate the practical effectiveness of EP estimation for ESL education.

\section{Acknowledgments}

This work was supported by JSPS KAKENHI (Grant Numbers 22300299, 15H02940, and 17K18679).

\section{References}

[1] Z. Gan, C. Leung, J. He, and H.-H. Nang, "Classroom assessment practices and learning motivation: A case study of Chinese EFL students," TESOL Q., vol. 53, no. 2, pp. 514-529, 2018.

[2] W. Grabe and X. Jiang, "Assessing reading," in The Companion to Language Assessment, A. H. Kunnan, Ed., Hoboken, NJ, USA: Wiley-Blackwell, 2014, pp. 185-200.

[3] K. Kotani and T. Yoshimi, "Predicting English proficiency with read-aloud performance and linguistic difficulty of sentences," in Proc. Int. Technol., Educ. Develop. Conf. (INTED2021), Mar. 8-9, 2021, pp. 1180-1185. Available: https://library.iated.org/publications/INTED2021

[4] Y. Lee, "Quantifying English fluency in Korean speakers' read-aloud and picture-cued storytelling speech," Linguist. Rev., vol. 31, no. 3, pp. 465-490, 2014.

[5] Handbook of the International Phonetic Association: A Guide to the Use of the International Phonetic Alphabet, Cambridge University Press, Cambridge, UK, 1999.

[6] D. Deterding, "The north wind versus a wolf: Short texts for the description and measurement of English pronunciation," J. Int. Phon. Assoc., vol. 36, no. 2, pp. 187-196, 2006.

[7] TOEIC Technical Manual, Chauncey Group International, Princeton, NJ, USA, 1999.

[8] V. N. Vapnik, Statistical Learning Theory. New York, NY, USA: Wiley, 1998.

[9] J. S. Chall and H. E. Dial, "Predicting listener understanding and interest in newscasts," Educ. Res. Bull., vol. 27, no. 6, pp. 141-153, 1948.

[10] A. Stenton, "The role of the syllable in foreign language learning: Improving oral production through dual-coded, sound-synchronised, typographic annotations," Lang. Learn. Higher Educ.: J. Eur. Confed. Lang. Centres Higher Educ., vol. 2, no. 1, pp. 145-161, 2013.

[11] I. E. Fang, "The easy listening formula," J. Broadcast., vol. 11 , no. 1 , pp. 63-68, 1966.

[12] H. Kiyokawa, "A formula for predicting listenability: The listenability of English language materials 2," Wayo Women's Univ. Lang. Lit., vol. 24, pp. 57-74, 1990.

[13] D. Meyer, et al., e1071: Misc Functions of the Department of Statistics (Formerly: E1071), TU Wien. Accessed: November 11, 2021. [Online]. Available: https:// cran.r-project.org/web/packages/e1071/e1071.pdf

[14] Y. Benjamini and Y. Hochberg, "Controlling the false discovery rate: A practical and powerful approach to multiple testing," J. Royal Stat. Soc. Series B (Methodological), vol. 57, no. 1, pp. 289-300, 1995.

[15] Institute for International Business Communication, "TOEIC program data \& analysis 2012." Accessed: June 16, 2021. [Online]. Available: http://www.toeic.or.jp/ library/toeic_data/toeic_en/pdf/data/TOEIC_Program DAA2012.pdf 